\title{
Water Diffusion in Complex Systems Measured by PGSE NMR Using Chemical Shift Selective Stimulated Echo: Elimination of Magnetization Exchange Effects
}

Accepted Manuscript: This article has been accepted for publication and undergone full peer review but has not been through the copyediting, typesetting, pagination, and proofreading process, which may lead to differences between this version and the Version of Record.

Cite as: J. Chem. Phys. (in press) (2021); https://doi.org/10.1063/5.0073704

Submitted: 03 October 2021 • Accepted: 14 November 2021 • Accepted Manuscript Online: 15 November 2021

Thomas G Meikle, (D) David W Keizer, (D) Frances Separovic, et al.

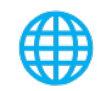

\section{Challenge us.}

What are your needs for periodic signal detection?

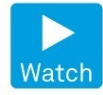

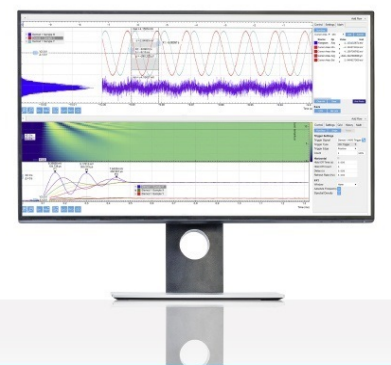

Zurich - Instruments 


\section{Water Diffusion in Complex Systems Measured by PGSE NMR Using Chemical Shift Selective Stimulated Echo: Elimination of Magnetization Exchange Effects}

Thomas Meikle", David W. Keizer², Frances Separovic ${ }^{2,3}$ and Shenggen Yao ${ }^{2, *}$

${ }^{1}$ School of Science, College of Science, Engineering and Health, RMIT University, Melbourne, VIC 3000, Australia

2Bio21 Molecular Science and Biotechnology Institute, The University of Melbourne, Victoria 3010, Australia

${ }^{3}$ School of Chemistry, The University of Melbourne, Victoria 3010, Australia

${ }^{*}$ Corresponding author:

Shenggen Yao

Bio21 Molecular Science and Biotechnology Institute

The University of Melbourne

Victoria 3010

Australia

Phone: +61 (3) 83442203

E-mail: shyao@unimelb.edu.au 


\section{ABSTRACT}

The interpretation of molecular translational diffusion as measured by pulsed gradient spinecho NMR (PGSE NMR) can be complicated by the presence of chemical exchange and/or dipolar cross-relaxation (including relayed cross-relaxation via spin diffusion). The magnitude of influence depends on the kinetics of exchange and/or dipolar cross-relaxation present within the system as well as the PGSE NMR sequences chosen for the measurements. Firstly, we present an exchange induced zero-crossing phenomenon for signal attenuation of water in lipidic cubic phases (LCPs, formed by a mixture of monoolein and water) in the presence of pulsed gradients observed using a standard STimulated Echo (STE) sequence. This magnetization exchange induced zero-crossing phenomenon, a pseudo negative diffractionlike feature, resembles that reported previously for restricted diffusion when locally anisotropic pores are polydisperse or randomly oriented. We then demonstrate the elimination of these exchange and/or dipolar cross-relaxation induced effects with the use of a chemical shift selective STE (CHESS-STE) sequence, adapted from the previously reported band-selective short transient STE (BEST-STE) sequence, along with results obtained from the bipolar pulse pair STE (BPP-STE) sequence for comparison. The CHESS-STE sequence introduced here represents a generic form of PGSE NMR sequences for obtaining water diffusion coefficients free from influence of exchange and/or dipolar cross-relaxation in complex systems. It has potential applications in measuring translational diffusion of water in biopolymer mixtures as well as probing microscopic structure in materials via water restricted diffusion measured by PGSE NMR, particularly when the potential presence of exchange/cross-relaxation is of concern.

KEYWORDS: Chemical shift selective sequence, CHESS-STE, Lipidic cubic phases, Magnetization exchange, PGSE NMR, Translational diffusion 


\section{INTRODUCTION}

Pulsed gradient spin-echo NMR (PGSE NMR) has established itself as a method of choice for the characterisation of molecular translational motion with applications ranging from spectroscopic analysis of complex mixtures ${ }^{1}$ to the quantitative analysis of protein folding or ligand binding ${ }^{2}$ and beyond, such as in magnetic resonance imaging (MRI). ${ }^{3}$ It has also seen broader applications in studying diffusive properties of water in aqueous (bio)polymer mixtures, such as hydrogels, and for evaluating the microscopic structure of porous media and emulsion systems via the time-dependent self-diffusion of confined fluids, typically water. ${ }^{4}$ Concurrently, the effects of magnetization transfer, including chemical exchange on intermediate timescales and/or dipolar cross-relaxation, in PGSE NMR has also been well investigated. ${ }^{5,6}$ With the exception of fast exchange where translational diffusion is degenerate and gives rise to a population weighted average value over sites involved in exchange, a full analysis of PGSE NMR data, under favourable conditions, may yield the kinetic parameters of exchange and/or cross-relaxation present in the system. On other occasions, when this exchange and/or cross-relaxation induced feature in PGSE NMR is unwanted, e.g. in exploring microscopic structure via restricted diffusion of fluids, then suppression is generally deemed necessary in order to minimise the probability of compromising the resultant PGSE NMR data.

Among the various forms of PGSE NMR sequences commonly in use, longitudinal relaxation-based STimulated Echo (STE) sequences ${ }^{7}$ are generally considered to be superior to their transverse relaxation-based Spin Echo (SE) counterparts. ${ }^{8}$ The primary reasons are that STE is less susceptible to $J$-modulation of coupled spins and is able to achieve sufficient diffusion encoding via the use of relatively long diffusion time for systems where longitudinal relaxation time $\left(T_{1}\right)$ is significantly longer than transverse relaxation time $\left(T_{2}\right)$. For example, ${ }^{1} \mathrm{H} T_{1}$ of water is approximately 20 times longer than $T_{2}$ in lipidic cubic phases (LCPs) formed by monoolein (MO). The effects of exchange and/or cross-relaxation in PGSE NMR is, however, significantly more pronounced for $T_{1}$-based STE sequences than that in $T_{2}$-based SE sequences, where magnetization transfer from chemical exchange and/or dipolar crossrelaxation does not result in net magnetization transfer, i.e., the effect of magnetization transfer is incoherent.

While molecular diffusion measured by PGSE NMR using nuclear spins other than protons are less likely to be susceptible to exchange and dipolar cross-relaxation, in practice they are generally considered to be less favourable due to reduced effectiveness in diffusion encoding and low signal sensitivity resulting from their relatively lower gyromagnetic ratio and natural abundance, i.e., their relatively rare occurrence in nature, when compared with protons. To a 
certain extent, these chemical exchange and/or dipolar cross relaxation induced effects in PGSE NMR may be circumvented by using a bipolar pulse pair of STE (BPP-STE) sequence ${ }^{9}$ when the effect of chemical exchange/cross relaxation effect during the diffusion encoding/decoding delays can be ignored. ${ }^{10,11}$ In particular, the practical effect of exchange in various forms of common PGSE NMR sequences, including SE, STE, LED (longitudinal eddy current delay) and BPP-LED sequences, has been evaluated previously. ${ }^{12}$ More specifically, dedicated forms of PGSE NMR sequences, which suppress exchange effects, have also been introduced, including: (i) periodic refocusing of J-evolution by coherence transfer (PROJECT) version of the spin-echo (SE) sequence; ${ }^{13}$ and (ii) an LED version of STE with $T_{2}$-filters designed for systems with significantly different transverse relaxation times between various compartments involved in exchange, such as a macromolecule and water. ${ }^{14}$

Frequency-selective RF pulses have become an indispensable component in modern NMR spectroscopy, such as for the simplification of NMR spectra of complex systems ${ }^{15}$ and for achieving spatial localization in MRI. In addition, selective RF pulses are commonly used for suppressing chemical shift artefacts or misregistration along the frequency encoding dimension in MRI, where the acronym CHESS (chemical shift selective) imaging was first introduced. ${ }^{16}$ Dedicated PGSE NMR sequences involving the use of frequency-selective or band-selective RF pulses have also been reported with various applications for which a specific spectral region or certain types of spins (such as amide protons) in the system is of interest. ${ }^{17,18}$ PGSE NMR measurements involving the use of frequency-selective RF pulses for supressing the influence of magnetization transfer from water to amide protons have previously been applied to studies of residue-specific $\mathrm{NH}$ exchange rates. ${ }^{19}$ In principle, when only translational diffusion of a single species or a unique type of spin within the systems is of interest, for example water, a generic solution to circumvent the problem of potential exchange and/or cross-relaxation is to only coherently perturb spins of interest in PGSE NMR measurements and leave all other spins either unperturbed or perturbed incoherently, such that they do not contribute coherently to the detected signals in terms of diffusion measurements.

Previously, we have explored several aspects of diffusion and exchange within LCPs. ${ }^{20,21}$ These materials, which form spontaneously in aqueous mixtures of lipids such as MO, feature two interpenetrating, but unconnected networks, of water channels, illustrated in Fig. 1. The resulting nanomaterials see applications in fields such as membrane protein crystallization and drug delivery, and serve as suitable model systems for probing diffusion and exchange effects within nanostructured systems. Depending on the level of hydration and the type of lipids used for the formation of LCPs, the water channels are typically several $\mathrm{nm}$ in diameter, and 
generally outside the size scale $\left(10^{-2}-100 \mu \mathrm{m}\right)$ of PGSE NMR for directly probing the nanostructure tortuosity via restricted/hindered diffusion of hydration water or soluble molecules encapsulated in the water channel. The hydration water and any solubilized molecules would, therefore, experience fully restricted diffusion, with the experimentally measured diffusion coefficient dependent, to a certain extent, on the tortuosity of the particular LCP under investigation, as described by its phase group and channel diameter or surface to volume ratio. ${ }^{4}$ In LCPs, water in the channel and that bound to the surface of the lipid bilayer undergo rapid exchange, on both the chemical shift and diffusion timescales accessible by PGSE NMR, and consequently the diffusion movement of water is degenerate and gives rise to a population weighted average value. Experimentally measured diffusion coefficients of water in LCPs by PGSE NMR are subsequently interpreted as the population-weighted average between unbound and bound fractions. ${ }^{22,23}$ PGSE NMR has been employed to measure diffusion properties of water, lipids and small drug-like molecules encapsulated in LCPs. ${ }^{20,22,}$ 24, 25 The correlations between the release profiles of model drug-like molecules from the LCP matrixes and their diffusion properties as measured by PGSE NMR have also been explored. ${ }^{20}$

In this report, we firstly present an exchange induced zero-crossing phenomenon, for signal attenuation of water diffusion in LCPs observed using the standard STE sequence. This exchange induced zero-crossing phenomenon, a pseudo negative diffraction-like feature, resembles that reported previously for restricted diffusion when locally anisotropic pores are polydisperse or randomly oriented. ${ }^{26-28}$ We then demonstrate the elimination of these exchange and/or dipolar cross-relaxation effects with the use of a chemical shift selective STE (CHESSSTE) sequence modified from our previously reported band-selective short transient STE (BEST-STE) sequence. ${ }^{17}$ Corresponding results obtained from a BPP-STE sequence are also included for comparison. ${ }^{9}$ The CHESS-STE sequence represents a generic form of PGSE NMR sequences for obtaining the diffusion coefficient of water, free from exchange and/or dipolar cross-relaxation influence, and should see potential applications in probing materials with microscopic structure via water restricted diffusion measured by PGSE NMR, where the potential presence of exchange/cross-relaxation is of concern. 

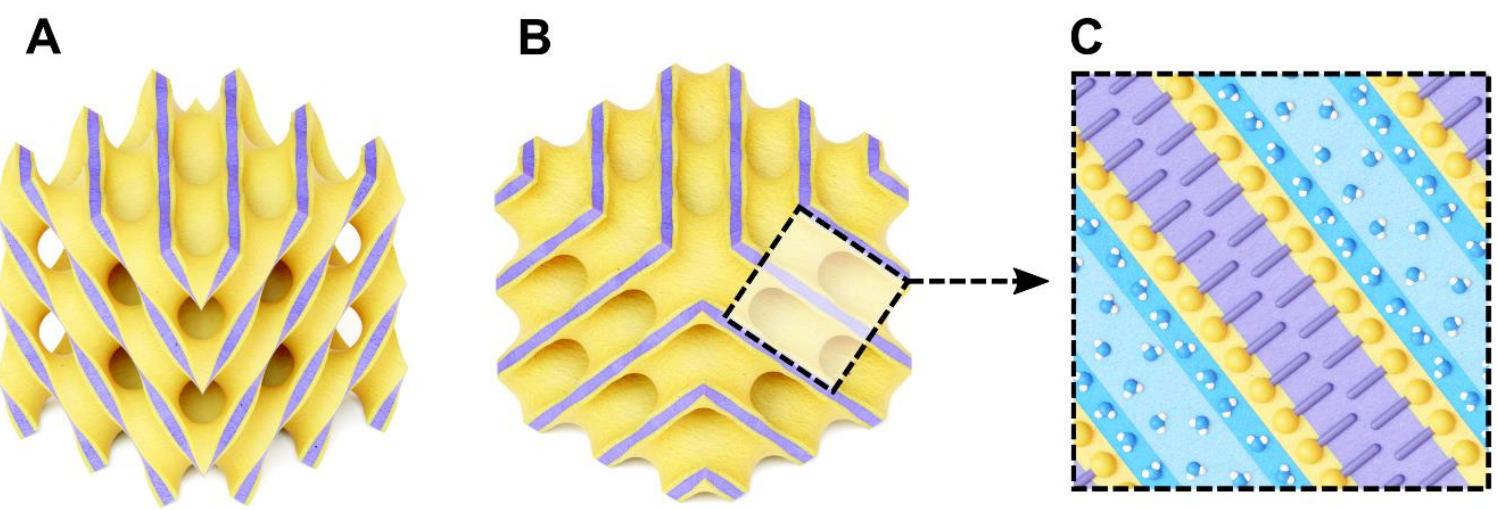

FIG. 1. A 3D illustration of the $Q_{\mathrm{II}}^{\mathrm{D}}$ LCP. (A) the lipid bilayer, with the hydrophobic region coloured purple and the head-group region coloured yellow, (B) the same $Q_{\mathrm{II}}{ }^{\mathrm{D}}$ LCP bilayer shown in (A) after a $180^{\circ}$ rotation around the vertical axis, and (C) a cross section of the LCP with the water channel highlighted in blue.

\section{MATERIAL AND METHODS}

\section{Sample preparation}

The LCP samples used in the present study were prepared by mixing molten monoolein (MO) (purchased from Sigma-Aldrich, St Louis, MO) with HEPES buffer solution, pH 7.0, at a hydration of $35 \mathrm{wt} \%$ using a coupled syringe apparatus as described previously. ${ }^{20}$ For NMR measurements, the LCP was injected into a glass capillary ( $\phi=2 \mathrm{~mm}$, o.d.), flame sealed, and subsequently placed into a standard $5 \mathrm{~mm}$ NMR tube filled with $\mathrm{CDCl}_{3}$, used to lock the magnetic field. ${ }^{21,29,30}$ A reference NMR sample containing just HEPES buffer in a sealed capillary tube surrounded by $\mathrm{CDCl}_{3}$ was also prepared.

\section{PGSE NMR data acquisition and analysis}

All PGSE NMR measurements were carried out on a Bruker Avance III $600 \mathrm{MHz}$ spectrometer, equipped with a TCI cryoprobe fitted with a single-axis field gradient $(G z)$ at $298 \mathrm{~K}$. The maximum gradient strength, $G_{\max }$, was calibrated to be $57.31 \mathrm{G} \mathrm{cm}^{-1}$ via back calculation against the published value of $1.90 \times 10^{-9} \mathrm{~m}^{2} \mathrm{~s}^{-1}$ at $298.13 \mathrm{~K}$ for the self-diffusion coefficient of residual $\mathrm{H}_{2} \mathrm{O}$ in a $100 \%{ }^{2} \mathrm{H}_{2} \mathrm{O}$ sample ${ }^{31}$ as described previously. ${ }^{32-34}$ A series of 32 spectra were acquired in a pseudo-2D mode for each PGSE NMR dataset with the gradient strength ranging from 2.87 to $54.44 \mathrm{G} \mathrm{cm}^{-1}$. The effective durations of the pulsed gradients used are $2.865 \mathrm{~ms}$ and $1.432 \mathrm{~ms}$, for measuring diffusion of water in LCP and in the HEPES reference sample, respectively. The number of scans for each spectrum was 16 with a recycle delay of $2 \mathrm{~s}$ between scans preceded by 8 dummy scans for the establishment of magnetization 
equilibrium. Sinusoidal shaped gradient pulses were used throughout the study for diffusion encoding and decoding. Diffusion data were processed in TOPSPIN (Version 3.6, Bruker) and an exponential window function with a line broadening of $3 \mathrm{~Hz}$ was applied before Fourier transformation. Initial analysis was performed using the $T_{1} / T_{2}$ relaxation module in TOPSPIN with final analyses performed using SigmaPlot (Version 12.5, Systat Software Inc).

\section{RESULTS AND DISCUSSION}

\section{Chemical exchange and dipolar cross-relaxation between water and lipids in LCPs}

As observed in many other lipid/water systems, ${ }^{35,36}$ the high concentration of lipids in LCPs facilitates dipolar cross-relaxation between water and alkyl chain protons of lipids. This includes relayed cross-relaxation via spin diffusion, ${ }^{37}$ under relatively long mixing times, as is frequently encountered in PGSE NMR when the accessible maximum strength of the field gradient is limited. In particular, the hydroxyl groups of the MO headgroup and water in LCPs undergo slow or intermediate exchange, as has been noted previously, ${ }^{21,38}$ and this effect has recently been further investigated via NMR exchange spectroscopy and PGSE NMR. ${ }^{21} \mathrm{~A}$ representative $2 \mathrm{D}{ }^{1} \mathrm{H}-{ }^{1} \mathrm{H}$ NOESY spectrum of LCPs formed by MO/water at $35 \mathrm{wt} \%$ hydration acquired with a long mixing time of $500 \mathrm{~ms}$, similar to those used in PGSE NMR measurements (see below), is depicted in Fig. 2. Cross peaks arising from exchange between water and MO hydroxyl groups as well as those originating from dipolar cross-relaxation between water and MO alkyl chain are clearly present. 
A
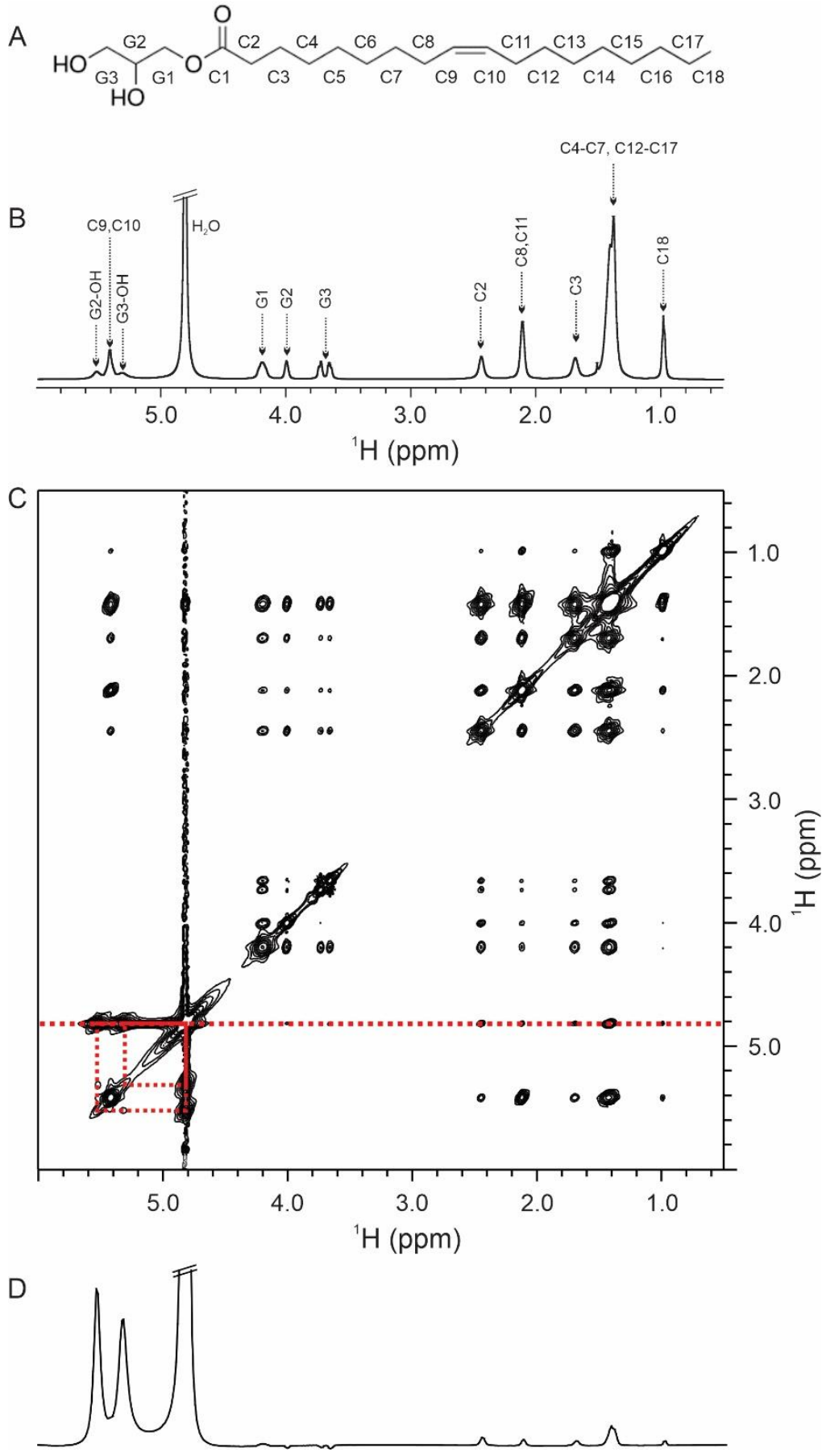

FIG. 2. Chemical exchange and dipolar cross-relaxation in LCPs formed by MO/water at 35 wt $\%$ hydration. (A) Chemical structure of MO with carbon atoms numbered sequentially, (B) 1D ${ }^{1} \mathrm{H}$ spectrum of $\mathrm{MO}$ with resonances labelled based on atom numbers depicted in (A), (C) 2D ${ }^{1} \mathrm{H}-{ }^{1} \mathrm{H}$ NOESY spectrum acquired using a mixing time of $500 \mathrm{~ms}$ with cross peaks arising from exchange between water and MO hydroxyl groups indicated in dashed boxes, and (D) 1D trace along the water chemical shift in F1 dimension (dotted line in red in (C)) highlighting cross peaks arising from (relayed) dipolar cross-relaxation between water and MO alkyl chain protons. 


\section{Water diffusion in LCPs measured by STE in the presence of exchange and/or dipolar cross-relaxation}

The presence of exchange and/or dipolar cross-relaxation in the system, however, may complicate the interpretation of the results obtained by PGSE NMR. For example, in microscopic structure studies of biporous materials by PGSE NMR, anomalous diffusion time, $\Delta$, dependence of the slow component of signal decay has been attributed to exchange processes between fluid in the primary and secondary pores. ${ }^{39}$ The translational diffusion induced signal attenuation of water in LCPs, formed by MO at $35 \mathrm{wt} \%$ hydration, in the presence of field gradients obtained using the standard STE sequence, is shown in Fig. 3A. A characteristic zerocrossing phenomenon is clearly evident in the $q$-space presentation (similar zero-crossing phenomenon is also present in conventional $K^{2}$, as defined in Eq. 1 (see below), space plots, data not shown) under the current experimental settings, i.e., with an effective gradient pulse width (defined as the product of the shape factor and the duration of the gradient pulses) of $2.865 \mathrm{~ms}$, a $\tau$ value of $4.7 \mathrm{~ms}$ (see schematic diagrams of pulse sequences shown in Fig. 4B) and diffusion time, $\Delta$ of $300,500,700 \mathrm{~ms}$. Similar zero-crossing (negative diffraction) phenomenon, considered analogous to diffusion-diffraction minima, for signal attenuation in the presence of pulsed gradients have been reported previously for locally anisotropic pores that are polydisperse or randomly oriented with a double-pulsed-gradient. ${ }^{26-28,40}$ For comparison, results obtained from a reference sample containing only the buffer solution, where zero-crossing phenomenon is not present, is also included in Fig. 3A. 

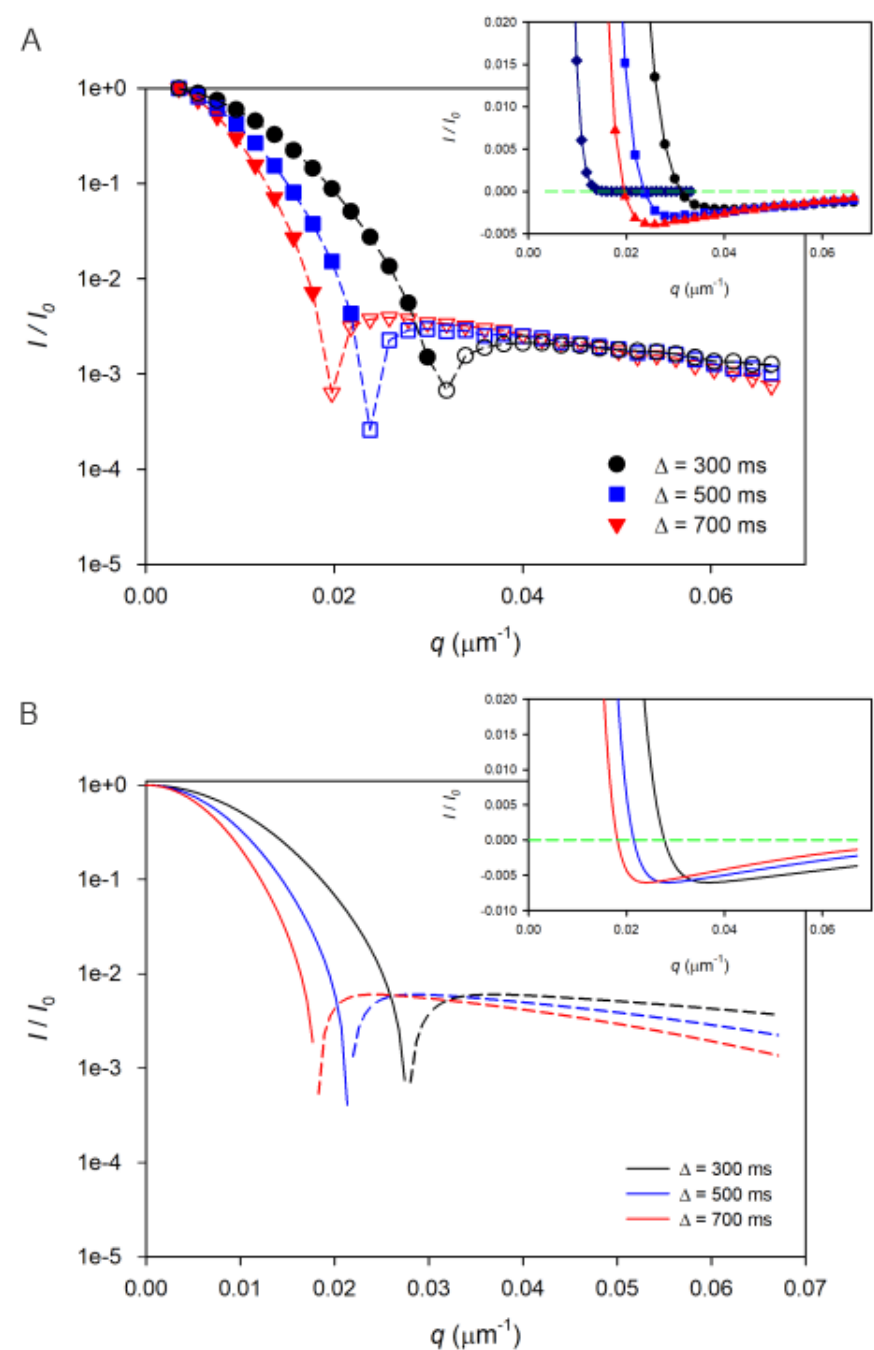

FIG. 3. A q-space presentation of magnetization exchange induced zero-crossing feature (negative diffraction) of signal attenuation in the presence of field gradient observed using STE sequence. (A) Signal attenuation of water in LCPs, formed by MO at $35 \mathrm{wt} \%$ hydration, in the presence of pulsed gradients acquired with an effective duration of gradient pulses, $\delta$ e, of 2.865 $\mathrm{ms}$ and a diffusion time, $\Delta$, of $300 \mathrm{~ms}(\bullet), 500 \mathrm{~ms}(\boldsymbol{\bullet}), 700 \mathrm{~ms}(\boldsymbol{\nabla})$. Filled symbols represent amplitude of positive water signal whereas open symbols represent absolute values of negative water signal. A negative diffraction-like feature is clearly present for all three diffusion times. Lines in the plots serve as a visual guide. A zero-crossing feature is clearly visible in the linear scale plot as shown in the inset. A similar plot for water signal of a reference HEPES buffer sample $(\diamond)$, acquired using an effective duration of gradient pulse, $\delta$, of $1.432 \mathrm{~ms}$ and a diffusion time, $\Delta$, of $500 \mathrm{~ms}$, is also shown in the inset, where zero-crossing is not present and the line represents the outcome of nonlinear regression using the standard Stejskal-Tanner expression, Eq. 1. (B) Simulated signal attenuation of water involved in two-site exchange based on Eq. 2. The following input parameters, close to those found in LCPs formed by MO at $35 \mathrm{wt} \%$ hydration, were used in the simulation: population of water and lipid hydroxyl group 9 to 1 , the diffusion coefficients of water and lipids as $5.5 \times 10^{-10}$ and $1.4 \times 10^{-11} \mathrm{~m}^{2} \mathrm{~s}^{-1}$, respectively, and a frequency difference of $370 \mathrm{~Hz} .{ }^{21}$ All other pulse sequence related parameters are identical to those used for the measurements shown in (A) including a value of $\tau=4.7 \mathrm{~ms}$ (see Fig. 4B). 
Theoretical considerations for exchange and/or dipolar cross relaxation in PGSE NMR, limited to a two-site model, have been described extensively ${ }^{5,6,11,41}$. In particular, the Kärger equations $^{42,43}$ form the framework for extracting kinetic exchange parameters from PGSE NMR measurements where exchanges, including dipolar cross-relaxation (via spin diffusion), occur on an intermediate timescale. ${ }^{44-48}$ Given the presence of two hydroxyl groups and potential contributions from direct and relayed dipolar cross-relaxation in LCPs, the apparent zero-crossing, pseudo diffraction-like phenomenon of water signal attenuation in LCPs (Fig. 3A) should be considered as the collective outcome of multiple sites involved in magnetization exchange with water. It can, however, be understood phenomenologically via signal perturbation in the presence of two-site slow exchange as described previously. The signal attenuation of isolated molecules experiencing free diffusion is given by

$$
I=I_{0} \exp \left\{-\gamma^{2} g^{2} \delta_{e}^{2}\left(\Delta-\frac{\delta_{e}}{3}\right) D\right\}=I_{0} \exp \left(-K^{2} D\right)=I_{0} \exp \left\{-4 \pi^{2} q^{2}\left(\Delta-\frac{\delta_{e}}{3}\right) D\right\}
$$

where $\gamma$ is the gyromagnetic ratio of protons, and $g, \delta$ and $\Delta$ are the amplitude, effective duration and separation of the encoding and decoding gradient pulses, respectively. The effective duration of the gradient pulse, $\delta$, is the product of the duration of the gradient pulses and the shape factor. ${ }^{49}$ Conventionally, the signal attenuation in the presence of field gradients is analysed against $K^{2}$, and sometimes is termed as $K^{2}$ space analysis. In contrast, when signal attenuation is inspected against the reciprocal lattice wave vector, $q\left(=\left(\frac{1}{2 \pi}\right) \gamma g \delta_{e}\right)$, commonly adopted in studies of restricted diffusion by PGSE NMR, it is termed as $q$-space approach first introduced by Callaghan et al. ${ }^{50}$ Significantly, in the $q$-space approach, a diffusion diffractionlike pattern may be present for signal attenuation of fluids, typically water, when sufficiently higher $q$-values are attained. The diffraction troughs, in the $q$-space approach, are then correlated to the microscopic dimension directly. For molecules involved in a two-site slow exchange, the signal attenuation of component $A$, for example water, can be written as follows, by taking into account the contribution from component $B:^{10,11}$

$$
\begin{gathered}
I=\rho_{a} \exp \left(-K^{2} D_{a}\right)+\rho_{b} \exp \left(-K^{2} D_{b}\right) \cos \left[\left(\omega_{a}-\omega_{b}\right) \tau\right] \\
=\rho_{a} \exp \left\{-4 \pi^{2} q^{2}\left(\Delta-\frac{\delta_{e}}{3}\right) D_{a}\right\}+\rho_{b} \exp \left\{-4 \pi^{2} q^{2}\left(\Delta-\frac{\delta_{e}}{3}\right) D_{b}\right\} \cos \left[\left(\omega_{a}-\omega_{b}\right) \tau\right] \\
\rho_{a}+\rho_{b} \equiv 1
\end{gathered}
$$

where $\rho_{\mathrm{a}}$ and $\rho \mathrm{b}$ are relative populations of component $A$ and $B$, respectively, and $\tau$ is the time between the first and second RF pulses (or between the third RF pulse and the centre of STE, see Fig. 4B). When either $\tau$ is sufficiently short or the chemical shift difference, $\left(\omega_{a}-\omega_{b}\right)$, is 
substantially small, the term $\cos \left[\left(\omega_{a}-\omega_{b}\right) \tau\right]$ approaches unity and Eq. 2a then reduces to a more commonly adopted biexponential form for signal attenuation of molecules involved in two-site slow exchange in PGSE NMR. ${ }^{51}$ As can be seen from Eq. 2a, depending on the relative population distribution and chemical shifts of site $A$ and $B$, as well as the $\tau$ value used in the measurements, the overall signal of the monitoring spins could turn out negative. As far as water diffusion is concerned in LCPs formed by MO at $35 \mathrm{wt} \%$ hydration, the population of water and hydroxyl groups in approximately 9 to1. The presence of an exchange induced zerocrossing, pseudo negative diffraction-like feature is expected at high $q$ values when contributions from slow diffusion component of lipids become significant. Fig. 3B shows simulated signal attenuations of water involved in a two-site exchange (Eq. 2) using population distribution of LCPs formed by MO at $35 \mathrm{wt} \%$ hydration, diffusion coefficients of water and lipids reported previously ${ }^{21}$ and chemical shift difference similar to that of hydroxyl groups and water (Fig. 2). With the same parameter settings for STE as used for measuring water diffusion in LCPs as shown in Fig. 3A, the characteristic zero-crossing phenomenon is clearly predicted by Eq. 2 .

\section{Chemical shift selective STE (CHESS-STE) sequence and elimination of magnetization exchange effects of water diffusion in complex systems}

For a given system consisting of a single major species or specific spin type, $W$ (e.g. water), slow or intermediate exchange induced effects on species $W$ in PGSE NMR measurements can be removed via the selective encoding and decoding of species $W$ together with the use of frequency-selective RF pulses in the presence of pulsed gradients. Various PGSE NMR sequences involving the use of frequency-selective RF pulses have been reported, with applications in systems where the translational motion of a certain group of spins (such as amide protons) or resonances are clustered into a sub-section of the spectrum (e.g. the upfield aliphatic region) is of interest. ${ }^{17,18}$ Several heteronuclear single quantum correlation (HSQC)edited sequences involving the use of frequency selective RF pulses for PGSE NMR have also been reported. ${ }^{52-54}$ A chemical shift selective STE (CHESS-STE) sequence together with the conventional STE and BPP-STE sequences used in the present study are depicted in Fig. 4. The CHESS-STE sequence was adapted from the BEST-STE sequence ${ }^{17}$ by replacing the first band selective $90^{\circ}$ pulses with a water selective $270^{\circ}$ Gaussian-shaped pulse with a width of $80 \mathrm{~ms}$ (commonly used for water suppression in biological NMR), corresponding to an excitation bandwidth of about $\sim 20 \mathrm{~Hz}$. The second $90^{\circ}$ pulse is EBURP-2 ${ }^{55}$ with a width of 
$2.42 \mathrm{~ms}$ (corresponding to an excitation bandwidth of about $\sim 2.0 \mathrm{kHz}$ ), and the third $90^{\circ}$ pulses are polychromatic $\mathrm{PC} 9^{56}$ shapes of $3 \mathrm{~ms}$ (corresponding to an excitation bandwidth of about $1.9 \mathrm{kHz}$ ) as previously used in BEST-STE. Offsets for all shaped-pulses were placed at water resonance (4.87 ppm in the present study of LCPs formed by MO and water mixture).
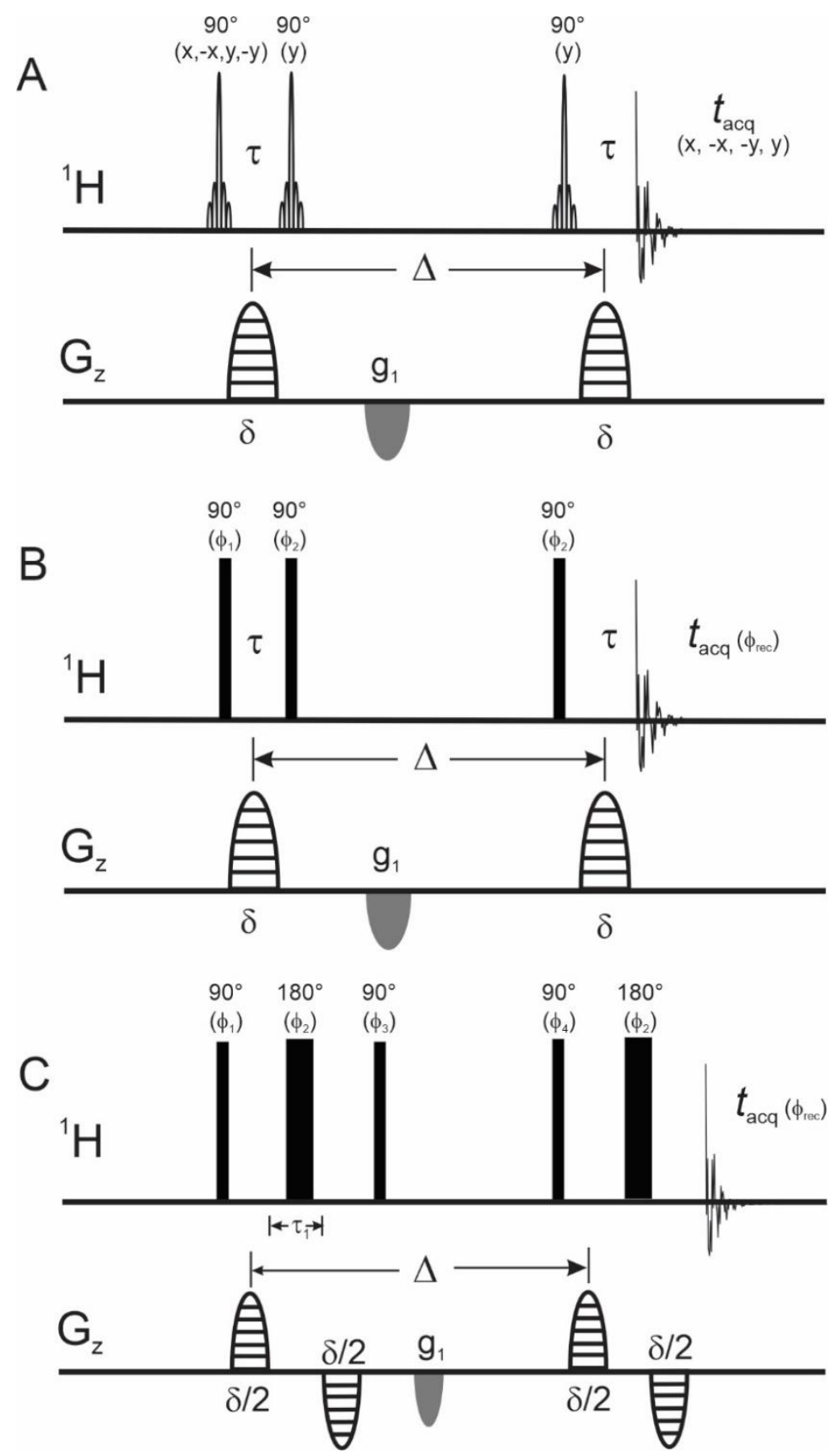

FIG. 4. Pulse sequences for measuring water diffusion in complex systems by PGSE NMR: (A) CHESS-STE, (B) standard STE, and (C) standard BPP-STE. In the present study, for CHESS-STE (A), the first selective $90^{\circ}$ pulse is a water selective $270^{\circ}$ Gaussian-shaped pulse with a width of $80 \mathrm{~ms}$ (corresponding to an excitation bandwidth of about $\sim 20 \mathrm{~Hz}$ ). The second $90^{\circ}$ pulse is EBURP-2 with a width of $2.42 \mathrm{~ms}$ (i.e., an excitation bandwidth of about 2.0 $\mathrm{kHz}$ ) and the third $90^{\circ}$ pulses are polychromatic PC9 shapes of $3 \mathrm{~ms}$ (i.e., an excitation bandwidth of about $\sim 1.9 \mathrm{kHz}$ ) same as previously used in BEST-STE. Narrow and wide 
rectangles represent $90^{\circ}$ and $180^{\circ}$ pulses in the standard LED sequence (B) and BPP-STE (C). Phases of RF pulses used for CHESS-STE (A) are as indicated whereas for STE (B) and BPPSTE (C) phase cycling used in stepgpls and stebpgpls (Bruker pulse sequence library) were adopted. In all sequences, gradient pulses (sinusoidal-shaped), used for diffusion encoding and decoding, are marked with horizontal lines.

As stated in the introduction, when only translational diffusion of a single species or unique types of spins, such as water, within the system is of interest, a generic solution to circumvent the problem of potential exchange and/or cross-relaxation is to coherently perturb only spins of interest in PGSE NMR measurements and leave all other spins either unperturbed or perturbed incoherently, so that they do not contribute coherently to the signals detected in terms of molecular diffusion measurements. Depicted in Fig. 5 and analogous to Fig. 3A, is a comparison of water single attenuation in LCPs formed by MO at 35\% hydration, in the presence of pulsed gradients and recorded using all three forms of STE sequences. As can be seen, the exchange induced pseudo negative diffraction-like feature (alternatively, the zerocrossing phenomenon, see inset) present in the signal attenuation of water in LCPs measured using standard STE sequence, vanished when the CHESS-STE sequence was employed. Notably, as expected, BPP-STE minimises the effects of the exchange induced zero-crossing feature with substantially suppressed negative signal intensities. Generally, the presence of intermediate exchange, such as magnetization exchange via dipolar cross-relaxation, and its influence in PGSE NMR may be not as apparent as slow exchange and consequently has been overlooked. The CHESS-STE, as a generic solution to circumvent the problem of potential exchange and/or cross-relaxation problem, results in a translational diffusion coefficient free of influence of intermediate and slow exchange as it only coherently perturbs the spins of interest and leaves all other spins either unperturbed or perturbed incoherently and, hence, they do not contribute to the detected signals. 


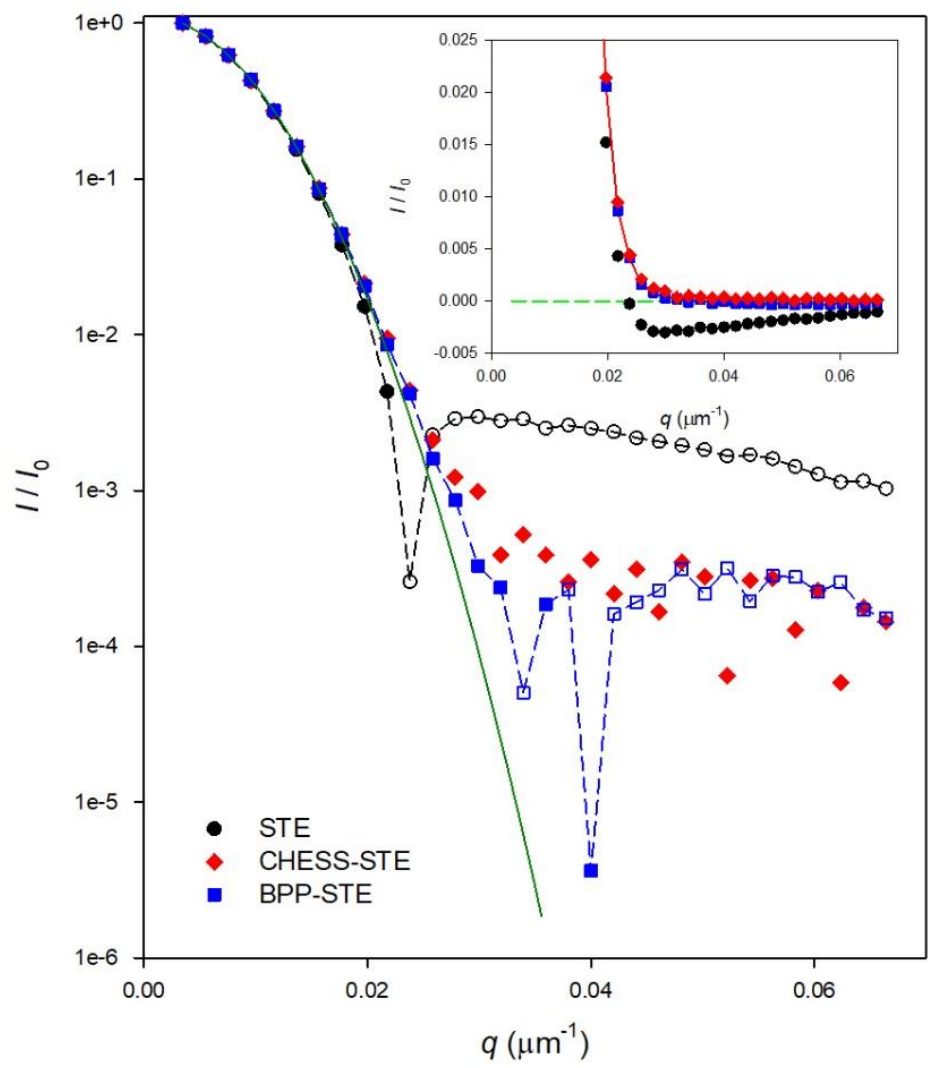

FIG. 5. Elimination of the magnetization exchange effect in PGSE NMR diffusion measurements using CHESS-STE. Signal attenuation of water in LCPs, formed by MO at 35 wt $\%$ hydration, in the presence of pulse field gradients acquired with STE $(\bullet)$, CHESS-STE $(\diamond)$, BPP-STE ( $\square$ ). Filled symbols represent the amplitude of positive water signal whereas open symbols represent absolute values of negative water signal. All data were acquired with an effective duration of gradient pulses, $\delta$, , of $2.865 \mathrm{~ms}(\tau=4.7 \mathrm{~ms})$, and a diffusion time, $\Delta$, of $500 \mathrm{~ms}$. The green line represents theoretical curve of diffusion in the absence of magnetization exchange calculated using Eq. 1, with parameters identical to those used in the experiments and the diffusion coefficient of water, $1.4 \times 10^{-11} \mathrm{~m}^{2} \mathrm{~s}^{-1}$, in LCP formed by $\mathrm{MO} /$ water at $35 \mathrm{wt} \%$ hydration. Magnetization exchange induced zero-crossing phenomenon, manifested by the presence of negative water signal values, vanished in data acquired with CHESS-STE. The line for data acquired with CHESS-STE $(\downarrow)$ shown in the inset represent the outcome of nonlinear regression using the standard Stejskal-Tanner expression, Eq. 1, with a fitted diffusion coefficient of $(1.135 \pm 0.001) \times 10^{-11} \mathrm{~m}^{2} \mathrm{~s}^{-1}$.

\section{CONCLUSIONS}

In summary, the presence of magnetization exchange may complicate the interpretation of molecular translational diffusion measured by PGSE NMR. When this exchange induced feature in PGSE NMR is undesirable, it needs to be suppressed in order to prevent the data being compromised. The CHESS-STE sequence described here produces a diffusion coefficient for spins virtually free of magnetization exchange effects, as long as the sites participating in magnetization exchange are resolved in the ${ }^{1} \mathrm{H}$ chemical shift dimension. This 
technique is expected to be beneficial in: (i) determining diffusion coefficient of water in agarose/water system or more generally water adsorbed to polymers, ${ }^{57-59}$ (ii) probing the microscopic structure of materials via water restricted diffusion measured by PGSE NMR, specifically where potential presence of exchange/cross-relaxation is of concern, and (iii) extracting exchange kinetic parameters by PGSE NMR via multiple-parameter nonlinear regression. ${ }^{48}$

\section{ACKNOWLEDGEMENTS}

The authors acknowledge support from the Bio21 Institute NMR Facility, University of Melbourne. S.Y. would like to thank Drs Jeffrey Babon (WEHI) and Marc-Antoine Sani (University of Melbourne) for helpful discussions.

\section{AUTHOR DECLARATIONS}

The authors have no conflicts to disclose.

\section{DATA AVAILABILITY}

The data that support the findings of this study are available from the corresponding author upon reasonable request.

\section{REFERENCES}

${ }^{1}$ I. J. Day, Prog Nucl Magn Reson Spectrosc 116 (2020) 1.

${ }^{2}$ A. Dehner, and H. Kessler, Chembiochem 6 (2005) 1550.

${ }^{3}$ Y. Cohen, and Y. Assaf, NMR Biomed 15 (2002) 516.

${ }^{4}$ P. N. Sen, Concepts Magn Reson A 23A (2004) 1.

${ }^{5}$ C. S. Johnson, J Magn Reson Ser A 102 (1993) 214.

${ }^{6}$ L. J. C. Peschier et al., J Magn Reson Ser B 110 (1996) 150.

7J. E. Tanner, J Chem Phys 52 (1970) 2523.

${ }^{8}$ E. O. Stejskal, and J. E. Tanner, J Chem Phys 42 (1965) 288.

${ }^{9}$ D. H. Wu, A. D. Chen, and C. S. Johnson, J Magn Reson Ser A 115 (1995) 260.

${ }^{10}$ A. D. Chen et al., J Am Chem Soc 120 (1998) 9094.

${ }^{11}$ S. V. Dvinskikh, and I. Furo, J Magn Reson 146 (2000) 283.

${ }^{12}$ L. Avram, and Y. Cohen, J Am Chem Soc 127 (2005) 5714.

${ }^{13}$ J. A. Aguilar et al., J Magn Reson 238 (2014) 16.

${ }^{14}$ G. Pages, S. V. Dvinskikh, and I. Furo, J Magn Reson 234 (2013) 35.

${ }^{15}$ H. M. Kessler, S.;Gemmecker, G., Magn Reson Chem 29 (1991) 527.

${ }^{16}$ A. Haase et al., Phys Med Biol 30 (1985) 341.

${ }^{17}$ S. Yao et al., Eur Biophys J 43 (2014) 331.

${ }^{18}$ P. W. Howe, Magn Reson Chem 55 (2017) 433.

${ }^{19} \mathrm{~T}$. Brand et al., J Magn Reson 187 (2007) 97.

${ }^{20} \mathrm{~T}$. G. Meikle et al., Nanoscale 9 (2017) 2471.

${ }^{21}$ T. G. Meikle et al., J Phys Chem B 125 (2021) 571. 
${ }^{22}$ P. O. Eriksson, and G. Lindblom, Biophys J 64 (1993) 129.

${ }^{23}$ O. Soderman, and P. Stilbs, Prog Nucl Magn Reson Spectrosc 26 (1994) 445.

${ }^{24}$ S. W. Jeong et al., Langmuir 18 (2002) 1073.

${ }^{25}$ T. J. Larkin et al., Magn Reson Chem 55 (2017) 464.

${ }^{26}$ E. Ozarslan, N. Shemesh, and P. J. Basser, J Chem Phys 130, 104702 (2009)

${ }^{27} \mathrm{~N}$. Shemesh et al., J Chem Phys 132, 034703 (2010)

${ }^{28} \mathrm{~N}$. Shemesh et al., J Chem Phys 133, 044705 (2010)

${ }^{29}$ T. G. Meikle et al., J Magn Reson 305 (2019) 146.

${ }^{30}$ T. G. Meikle et al., Langmuir 36 (2020) 6254.

${ }^{31}$ R. Mills, J Phys Chem 77 (1973) 685.

${ }^{32}$ P. T. Callaghan, M. A. Legros, and D. N. Pinder, J Chem Phys 79 (1983) 6372.

${ }^{33}$ S. Yao, G. J. Howlett, and R. S. Norton, J Biomol NMR 16 (2000) 109.

${ }^{34}$ S. Yao, J. J. Babon, and R. S. Norton, Biophys Chem 136 (2008) 145.

${ }^{35}$ D. Canet, N. Mahieu, and P. Tekely, J Am Chem Soc 114 (1992) 6190.

${ }^{36}$ D. Huster, and K. Gawrisch, J Am Chem Soc 121 (1999) 1992.

${ }^{37}$ A. Pampel et al., Chem Phys Lett 287 (1998) 468.

${ }^{38}$ E. Boyle-Roden et al., J Magn Reson 189 (2007) 13.

${ }^{39}$ P. V. Kortunov, and V. D. Skirda, Colloid Journal 67 (2005) 573.

${ }^{40} \mathrm{~N}$. Shemesh, and Y. Cohen, J Magn Reson 195 (2008) 153.

${ }^{41}$ E. J. Cabrita et al., J Magn Reson 157 (2002) 124.

${ }^{42}$ J. Karger, Adv Colloid Interfac 23 (1985) 129.

${ }^{43}$ D. Wijesekera et al., Concepts Magn Reson A 47a (2018)

${ }^{44}$ M. Schonhoff, and O. Soderman, J Phys Chem B 101 (1997) 8237.

${ }^{45}$ D. Topgaard, and O. Soderman, Langmuir 17 (2001) 2694.

${ }^{46} \mathrm{C}$. Wende, and M. Schonhoff, Langmuir 26 (2010) 8352.

${ }^{47}$ A. E. Frise et al., J Phys Chem B 116 (2012) 2635.

${ }^{48}$ L. Escobar et al., Chem-Eur J 26 (2020) 8220.

${ }^{49}$ W. S. Price, and P. W. Kuchel, J Magn Reson 94 (1991) 133.

${ }^{50}$ P. T. Callaghan et al., Nature 351 (1991) 467.

${ }^{51}$ S. Barhoum, S. Palit, and A. Yethiraj, Prog Nucl Magn Reson Spectrosc $94-95$ (2016) 1.

${ }^{52}$ R. Augustyniak et al., J Biomol NMR 50 (2011) 209.

${ }^{53}$ M. Shukla, and K. Dorai, J Magn Reson 213 (2011) 69.

${ }^{54}$ S. Yao et al., Eur Biophys J 47 (2018) 891.

${ }^{55} \mathrm{H}$. Geen, and R. Freeman, J Magn Reson 93 (1991) 93.

${ }^{56}$ E. Kupce, and R. Freeman, J Magn Reson Ser A 102 (1993) 122.

${ }^{57} \mathrm{~J}$. Tritt-Goc, and J. Kowalczuk, Langmuir 28 (2012) 14039.

${ }^{58} \mathrm{~J}$. Kowalczuk, J Mater Sci 52 (2017) 11101.

${ }^{59}$ H. Kojima et al., J Phys Chem B 125 (2021) 9357. 
A

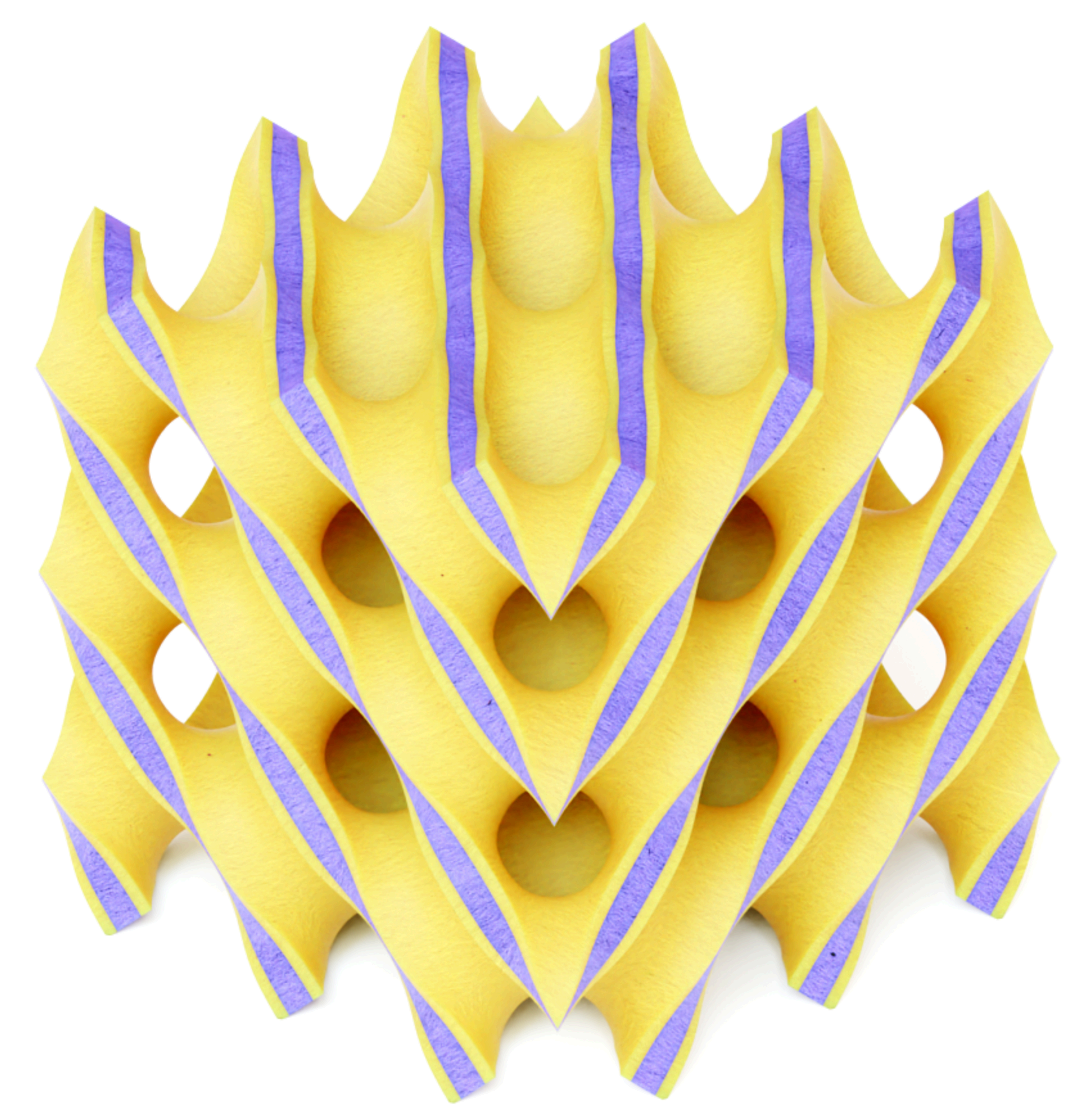

B

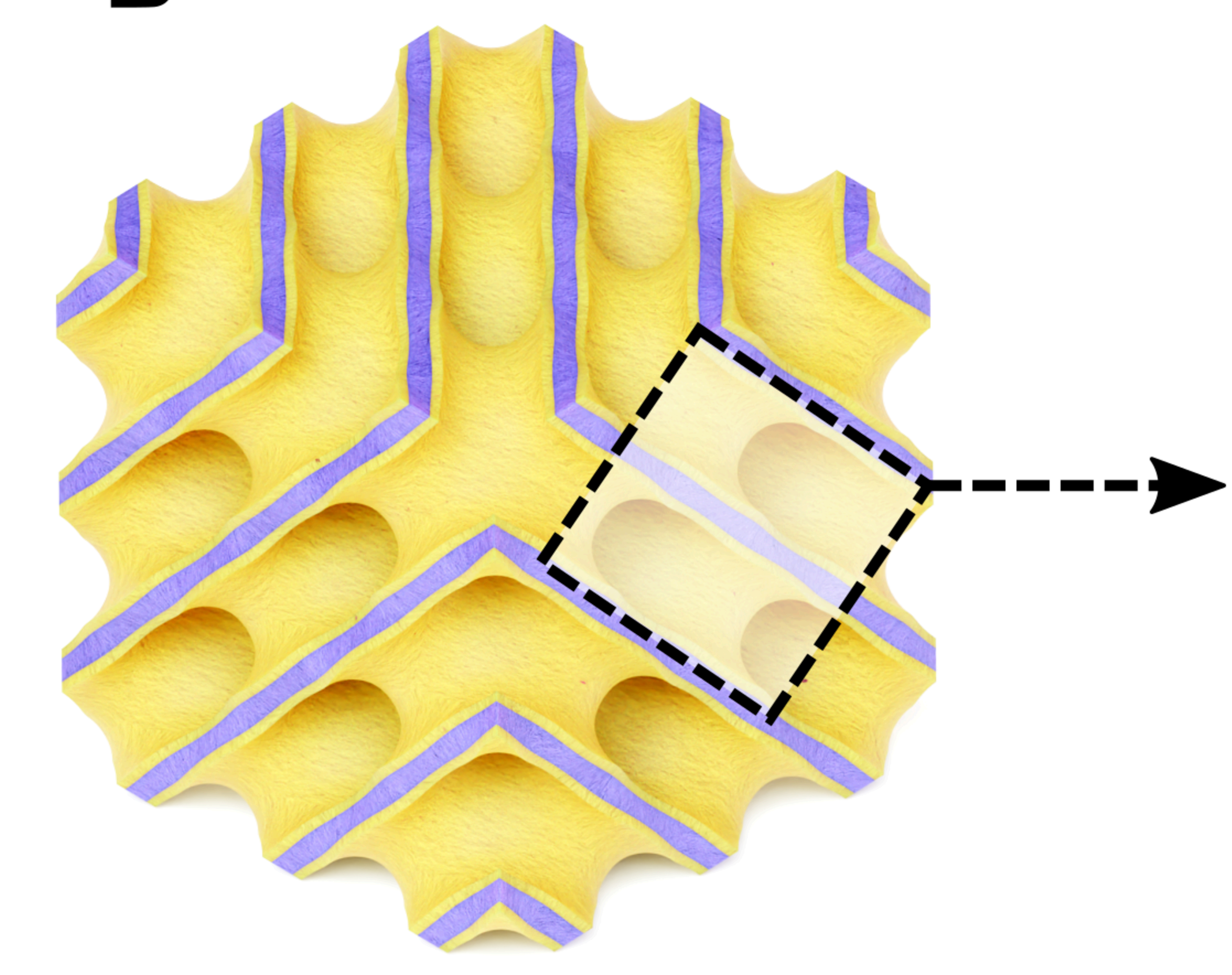

C

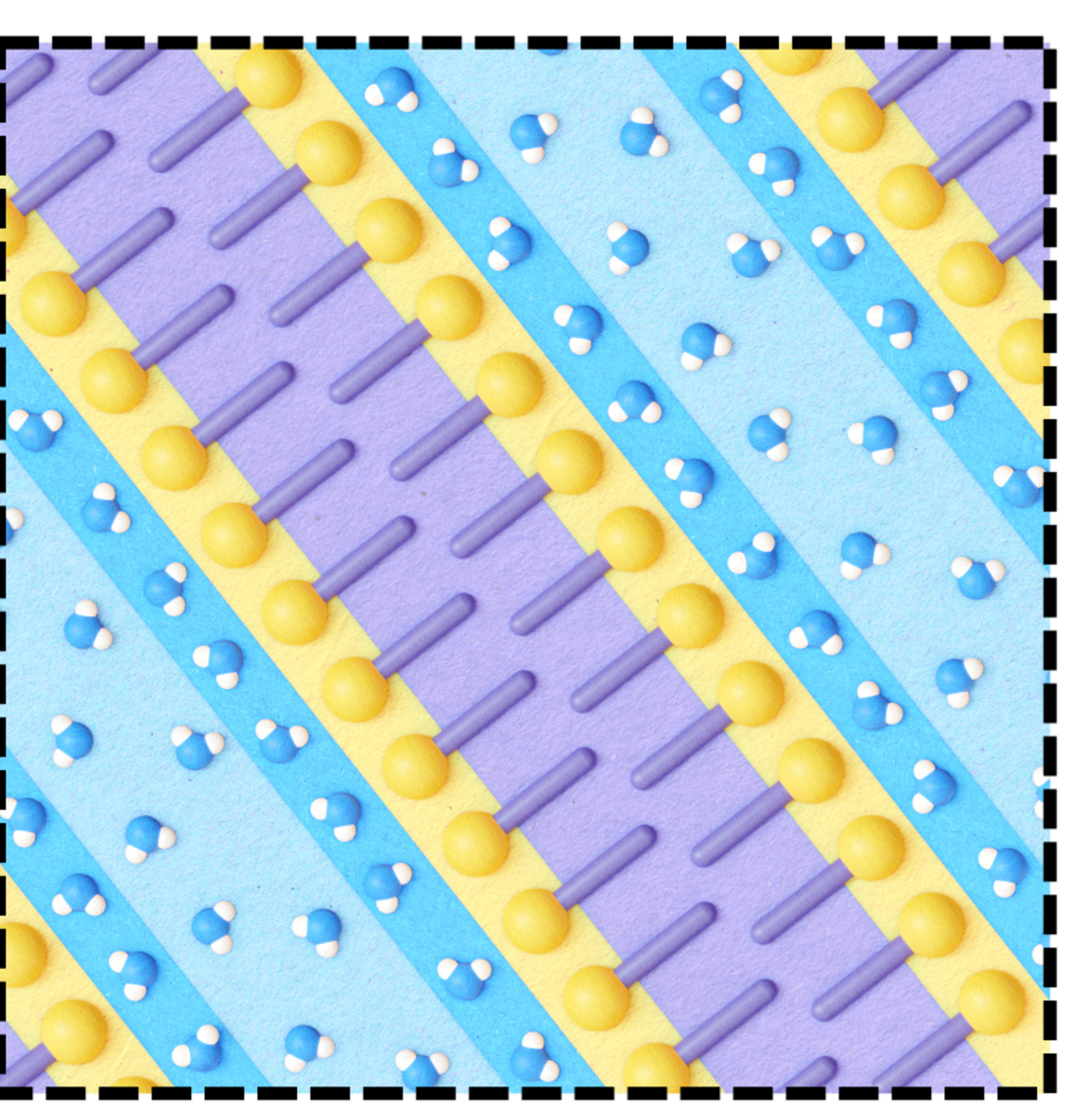


A

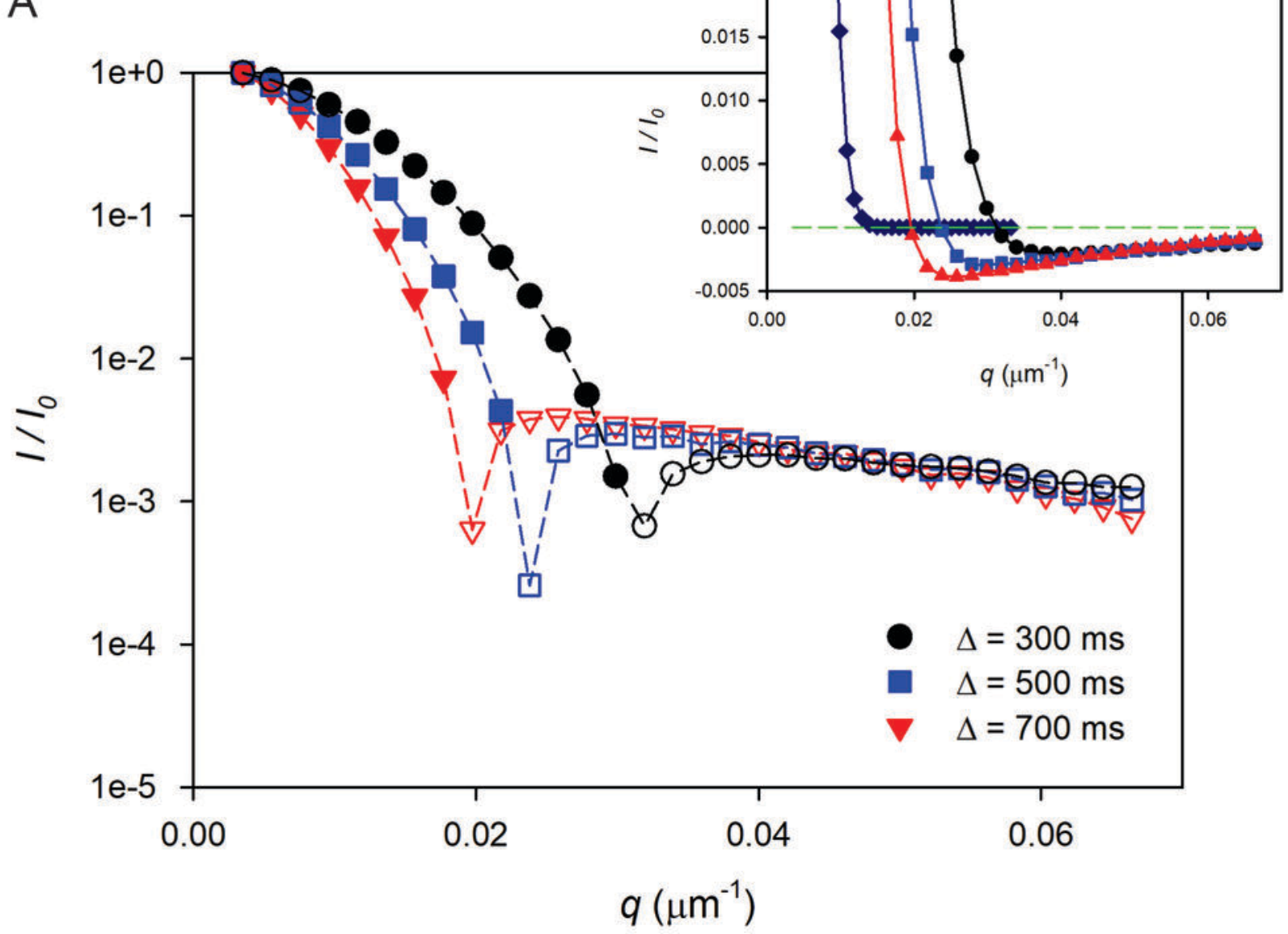

B

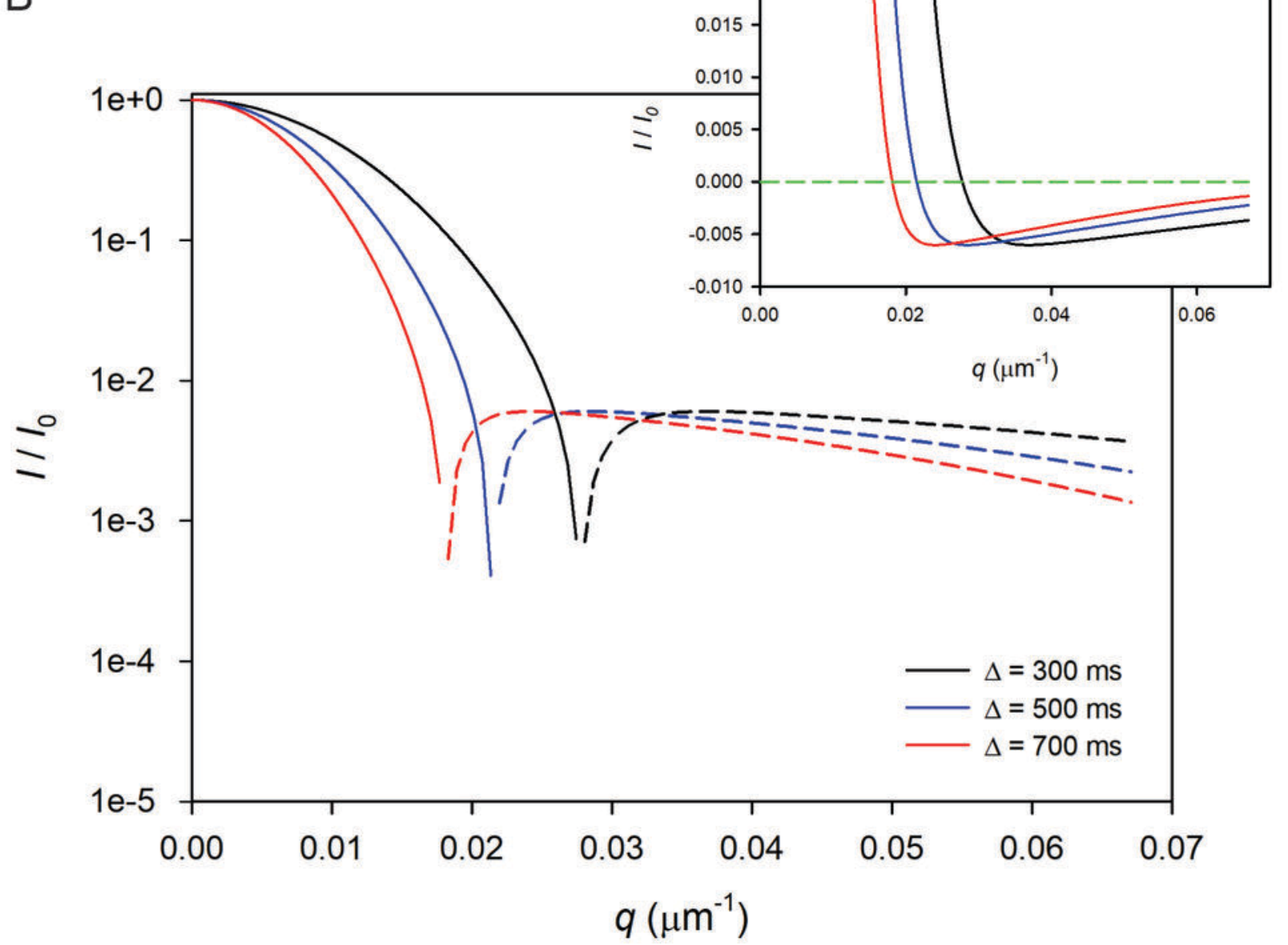


$A$

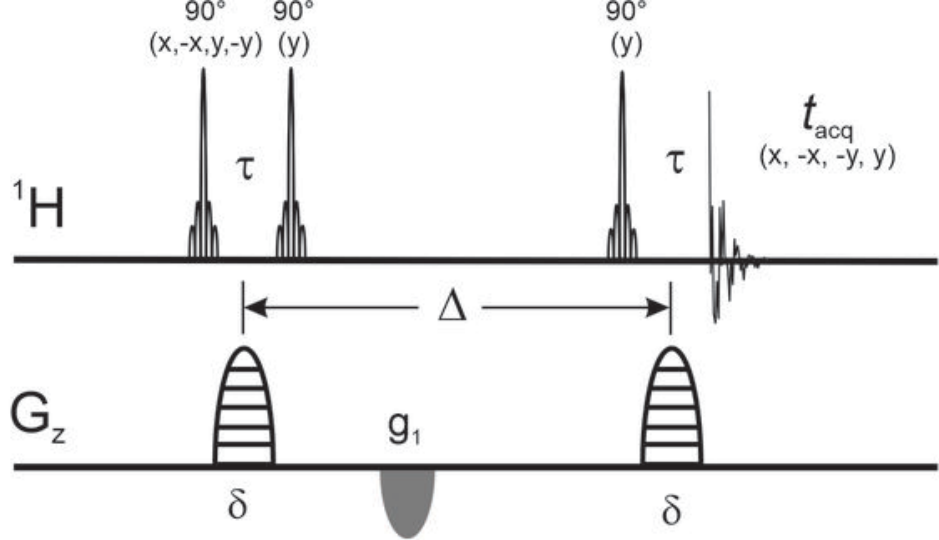

B
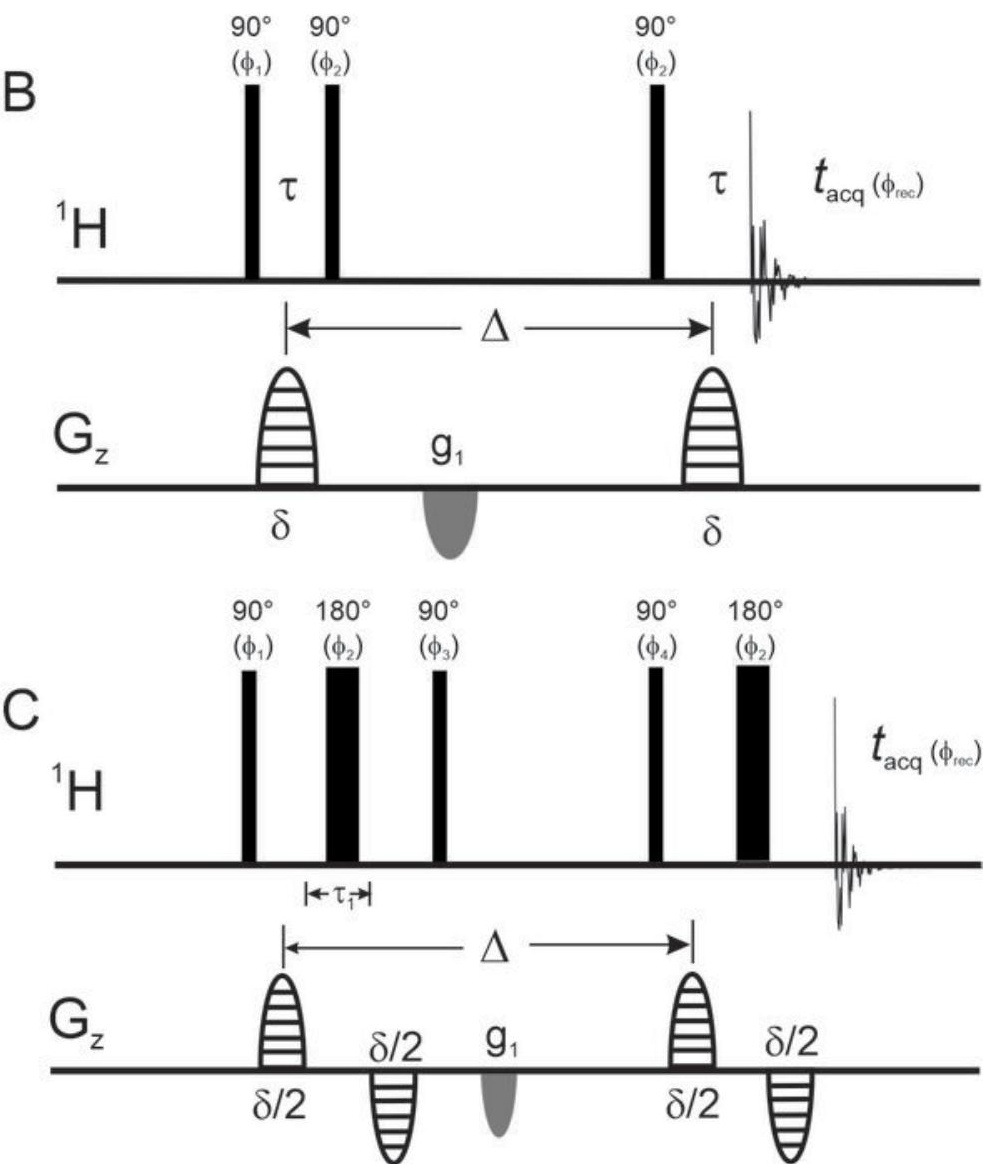
